\title{
Phase-Detection Research of Intelligent Substation based on embedded system
}

\author{
Yang Yongbo \\ State Grid Henan electric power company \\ Zhengzhou Henan ,China
}

\author{
Li Junhua, Wang Kun, Zhan Zhu, Wang Hezhe, \\ Gu Xiaodong \\ State Grid Henan electric power company \\ Zhengzhou Henan ,China
}

\begin{abstract}
With the rapid development of intelligent substation, GIS equipment and electronic transformer are widely used. So traditional phase-detection on high-pressure substation has been unable to achieve. Using virtual instrument technology, this paper designed a phase-detection meter on embedded computer systems, which uses embedded computer as the processor, and Visual $\mathrm{C}$ to develop the interface software. propose effective solutions. This meter is portable, universal, and secure, and this research has practical values.
\end{abstract}

Keywords-Intelligent Substation; Phase-Detection; Electronic Transformer; Embedded

\section{INTRODUCTION}

Since the expansion of the power transmission and transformation project, transform, and main equipment maintenance and repair, measuring the power phase is necessary before putting into operation, which is the phase-detection, in order to prevent different phase switching interphase short circuit, causing accident. phasedetection at the same time, to ensure that users in the dual power supply system using any of the power supply, motor "inverted" phenomenon doesn't occur, to ensure normal operation of electrical equipment[1].

Phase-detection includes checking phase sequence and phase, using special instruments directly to check the two which are on the closed loop system related to power supply voltage, or indirectly through the related device on the two systems, such as voltage transformer voltage, to contrast phase sequence and phase whether are the same.

In the construction of power engineering, phasedetection operation is a very important stage in the work. (1) In the new installation or maintenance main transformer substation (or voltage transformer, station transformer) in operation after the expansion, (2) the change of the external and internal wiring, or a set of transformer terminal, (3) New transmission lines connected to the transformer substation architecture, (4) the change of the circuit wiring or change the high-voltage electric power wire (or cable), often to the phase test.

\section{TRADITIONAL SUBSTATION PHASE-DETECTION}

Traditional methods for phase-detection in substation, the paper gives a brief introduction. Voltage with $110 \mathrm{kV}$ and below, if it is open outdoor equipment, or switchgear systems, with drain bare live parts in the two systems, we can use the corresponding level of high-pressure phasedetection instrument direct contact to detect phase. Voltage with $220 \mathrm{kV}$ and above systems, usually use indirect phasedetection methods such as the use of voltage transformer on the associated power[2,3].

\section{A. High-voltage direct phase-detection}

For system which voltage with $110 \mathrm{kV}$ and below, using high-pressure phase-detection meter directly test. Because the traditional high-pressure phase-detection meter with higher risk, now high-voltage wireless phasedetection meter is widely used.

Four high-pressure phase-detection simultaneously with the completion of the work required. One of them is as a conductor, and responsible for monitoring phasedetection remind staff to operate. As a member of the phase-detection two people need to wear insulated shoes, and wear insulated gloves, operating a high-pressure collector with an insulating rod. Need a man armed with a host monitor, meter reading results and record data. Phasedetection work should comply with an order commanding officer, member of the high-pressure phase-detection acquisition firmly secured in the insulating rod, insulation rod into contact with high-voltage power supply. The recorder records the measurement results. In order to ensure the reliability of the measurement results, measurements should be carried out several times, and by crossing measured phase by phase.

Outdoor open type device can easily be detected. Now $10 \mathrm{kV}$ system designed for multi-switch cabinet. To ensure safety, $10 \mathrm{kV}$ bus mostly closed-end, when the trolley pulled up to the test position, the mechanical shutter falling block stationary contacts. For this purpose the design of the phase-detection dedicated trolley, pushed by the phasedetection handcart, lifting $10 \mathrm{kV}$ switchgear bus baffle blocking function, the following procedures and outdoor equipment phase-detection procedure is the same.

\section{B. Indirect phase-detection}

For system which voltage with more than $220 \mathrm{kV}$, usually we can conduct a indirect phase-detection. The two systems will be sent to the two phase-detection voltage transformer or the transformer station, the phase difference detected by the phase-phase voltage. If different phase with phase lead or lag of $120^{\circ}$, the same description of two power phases.

1) The voltage transformer (VT) phase-detection

Firstly, the indirect wiring unanimously adopted two second phase-detection voltage transformer calibration method, the second phase is correct (for the substation has 
not changed through the check and wiring VT, can be omitted). That same power source to be located on the phase-detection two VT bus. Check with a voltmeter VT secondary output phase voltage of about $60 \mathrm{~V}$, and then use the phase sequence tables checking two VT low voltage phase sequence is correct, and finally at the same time check measuring two-phase low voltage VT output voltage difference is approximately zero, different phase voltage is approximately $100 \mathrm{~V}$. Above measurement requirements are met, then the two systems have the same phase, and it can be run in parallel. Otherwise, to analyze the specific case, check the wiring once again after the phase-detection.

\section{2) Phase multi-function meter method}

In the two systems with no VT groups of cases, the use of single-phase VT to test, but this method need to set up a separate test VT, which is less secure. Phase multifunction meter method can be employed by VT on both systems, the measured phase angle between the same fixed phase. When the same three-phase secondary phase angle difference may explain secondary wiring is correct. Phase meter method test equipment should ensure accurate recording, and analysis must be accurate.

\section{INTELLIGENT SUBSTATION PHASE-DETECTION}

Because electromagnetic resonance conventional electromagnetic transformers, saturated excitation and other characteristics, the intelligent electronic transformer substation has been applied. Through the optical fiber is converted to a measurement result of the transmission of digital signals to the merging unit (MU), and then through the process layer network traffic to the appropriate protection. Typical smart substation wiring is shown below:

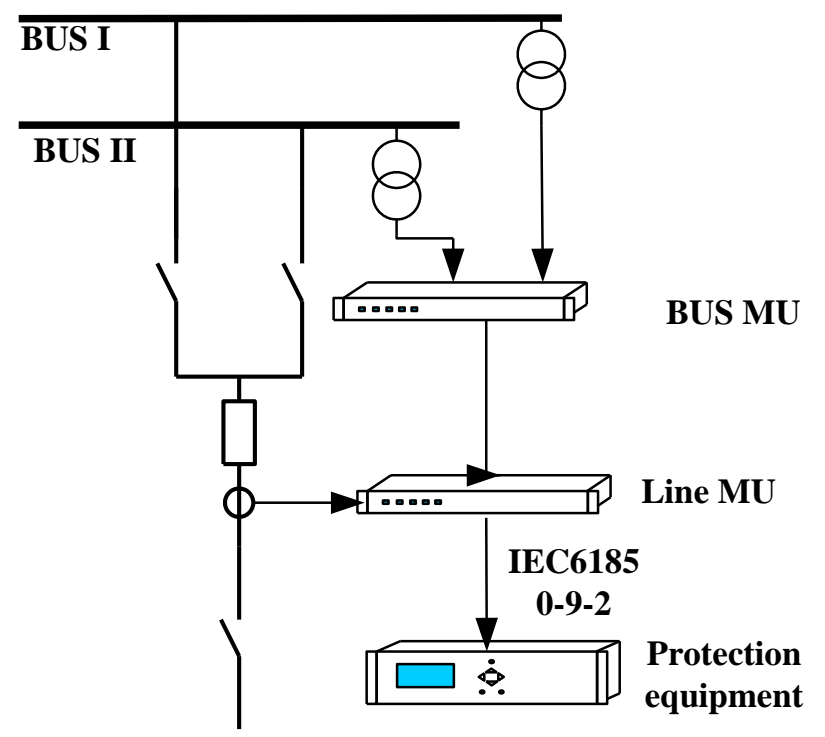

Figure 1. Intelligent substation secondary equipment Chart

At the same time, because of the wide application of GIS substation equipment, power transmission cable laying, a direct nuclear leak live naked with no part of the traditional phase-detection program has been unable to achieve. Therefore, it is necessary to analyze the voltage measurement system intelligent substation, and the corresponding phase-detection solution.

3.1 Intelligent Substation structure

Intelligent substation featuring "three two networks" structure to achieve the second network transmission equipment, by merging units will collect electronic transformer voltage and current data to the process layer networks for relevant IED devices.

Electronic transformer technology with the rapid development of electronic technology and optical fiber communications gained considerable progress. Compared with traditional transformers, electronic transformer has the following advantages:

- The high and low side isolation, insulation simple, cost-effective;

- the elimination of ferromagnetic resonance and magnetic saturation and other issues;

- Good resistance to electromagnetic interference, no risk of secondary coil open circuit;

- high precision, wide dynamic range, wide frequency response range.

Electronic transformer through optics, electromagnetic and other sensors, through the signal conditioning circuit converts the high voltage signal into a digital signal once the device, through the optical fiber transmission, supply of secondary devices. Meanwhile, the electronic transformer greatly reduce the amount of electricity using cables, the field devices simplify complex fiber optic cable routing and easy construction and installation.

MU interface device as a process-level voltage and current transformers and the spacer layer of the IED has an irreplaceable role. The main function is to collect light synchronous digital signal output of electronic transformer and sent to the protection and monitoring in accordance with uniform standards for data formats devices.

MU and electronic transformer interface includes three types: First, MU digital and electronic transformer between the serial data transmission, can be asynchronous (UART) transmission, but also can sync mode (Manchester coding) transmission. Second, MU input electronic transformer multi-mode fiber optic Ethernet module, using ST interface. Third MU laser and electronic transformer energizing interface.

3.2 Current intelligent substation phase-detection

When the traditional phase-detection implementation plan inconvenience, intelligent substation phase-detection program, mainly through live monitor phase-detection.

Live display principle phase-detection[4]. Line in substation, often equipped with live display, as an auxiliary electrical inspection measures, as well as blocking condition to provide anti-misuse by blocking logic. The use of live display principle, from the electrode side of the live display monitor dismantlement little secondary wiring connected to an oscilloscope or phase-detection meter, you can easily measure the phase sequence between the three phases. When phase-detection, the secondary side of the same name two lines of phase high pressure system brought by shielded cable to the oscilloscope or phasedetection meter, you can refer to PT second phasedetection-related technical regulations, the phase relationship between the two systems whether they have rings condition. The diagram of live display principle phase-detection as follows: 


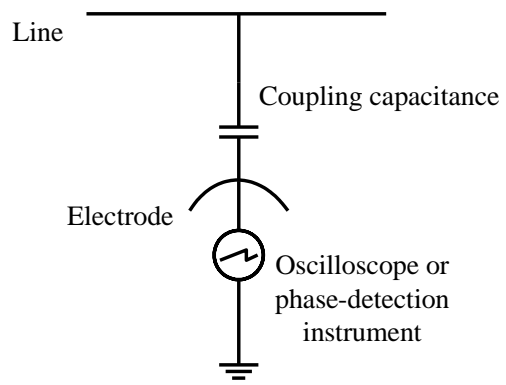

Figure 2. The diagram of live display principle phase-detection

Through live display principle phase-detection, we need to remove the cover from the cable compartment GIS voltage live display cited source, which affecting the primary safety device. Substation phase-detection work is usually only needed in new investment or expansion, renovation project with this large amount, resulting in unnecessary waste. Meanwhile, the live display space is used in the coupling capacitor, affecting vulnerable to electromagnetic interference space. In phase-detection process, it will lead to a phase-detection results accuracy is not high.

Such can not be applied on non-live display of two busbars phase-detection, with greater limitations, for which it is necessary to develop a portable, universal phase-detection meter[5].

\section{SYSTEM DESIGN}

System consists of signal acquisition modules, embedded host processing module and LCD touch screen and other peripheral circuits. Embedded host as the control and processing center systems, data processing storage and display graphical environment, the use of programming virtual instrument panel, complete the transfer of data, processing, storage, analysis, display and other functions. System components as shown below:

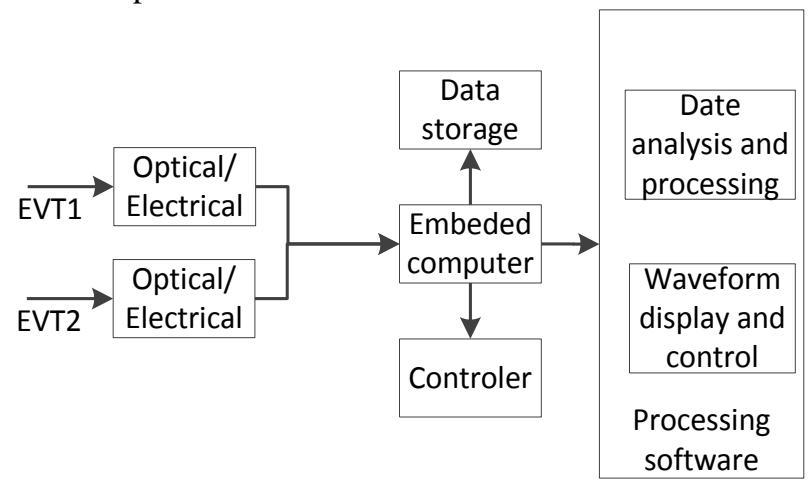

Figure 3. The overall block diagram of the system

The MU optical signal emitted by the photoelectric conversion element to transfer data measurement results to embedded computers, embedded computers merge unit packet parsing, extracting value signal for further analysis and processing. The system uses intelligent $10 / 100 \mathrm{M}$ adaptive fast Ethernet fiber optic transceivers. The fiber optic transceiver features are as follows: Full and half duplex auto-negotiation mode transmission. Twisted pair ports support auto-crossover, dual-fiber multi-mode, dualfiber single-mode and single-fiber single-mode fiber interface. Ultra Low power consumption (less than $2 \mathrm{~W}$ ), low heat, stable operation for a long time.
The peripheral circuit includes a touch screen and a power supply module. Touchscreen provides interactive features, control measurement and display of measurement results.

\section{A. Embedded Computer}

Embedded nature of the object is embedded computer system embedded in a computer, so it has a completely different direction of technology development and requirements. Reliability and the ability to target embedded control intelligent control system is the main technical direction of embedded computer systems. In addition to the intelligent design of embedded computer control, often with strict limitations and requirements, such as small size, light weight, low power consumption, safe and reliable, adaptability to harsh environments, sensitivity of cost. Therefore, the system must be designed according to the needs, considering, preferred choice of embedded computer.

Ultra-low-power embedded computer embedded CPU AMD Geode LX series. Schematic embedded computers are as follows, by AMD GEODE CS5536 auxiliary controllers embedded computer design interactive interface with the outside world, features include a hard disk interface, USB interface, PCI BUS, Ethernet port and Flash BIOS interface.

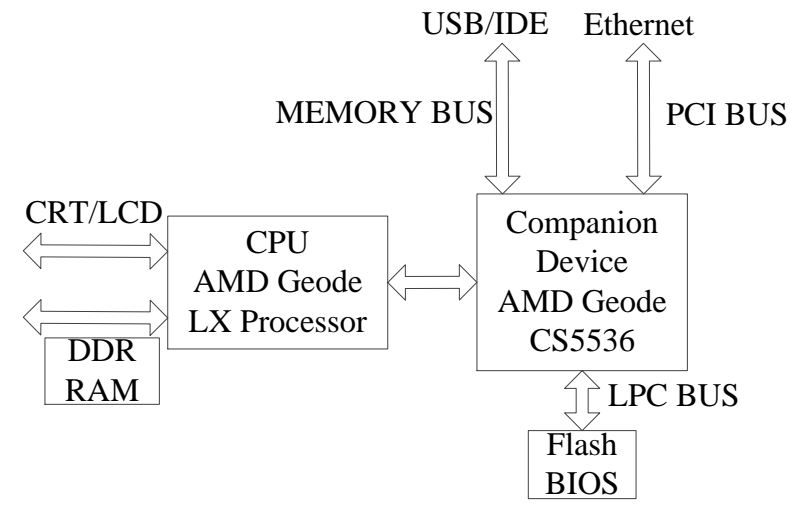

Figure 4. Embedded Computer Schematic

\section{B. System Software Design}

Embedded computer operating system available mainly VxWork, embedded Linux, Palm OS and Windows XPe. VxWork good real-time performance, in addition to support for embedded processor also supports external RISC, DSP technology, its system features a fast multitask switching and preemptive task scheduling. Embedded Linux is the result of the reduction of the Linux system. The kernel and the file system is very small, less than 900 $\mathrm{kB}$, but powerful, with a common Linux API and complete TCP / IP protocol stack. Since Palm OS has an open API (application program interface operating system) , developers may need to be developed according to the needs of the program.

In this paper, the development of embedded systems, using the Windows XP Embedded facilitate the use of data acquisition card driver and control procedures. Windows XP Embedded operating system has all the features and attributes of Windows XP Professional, and Professional compatible application software, but also has Windows CE system software requirements can be based on the 
characteristics of any reductions, while less system kernel small, system resources, fast start The system has enhanced write-protected.

Due to the modular hardware system, universal, system software design is especially important. Software process structure shown below.

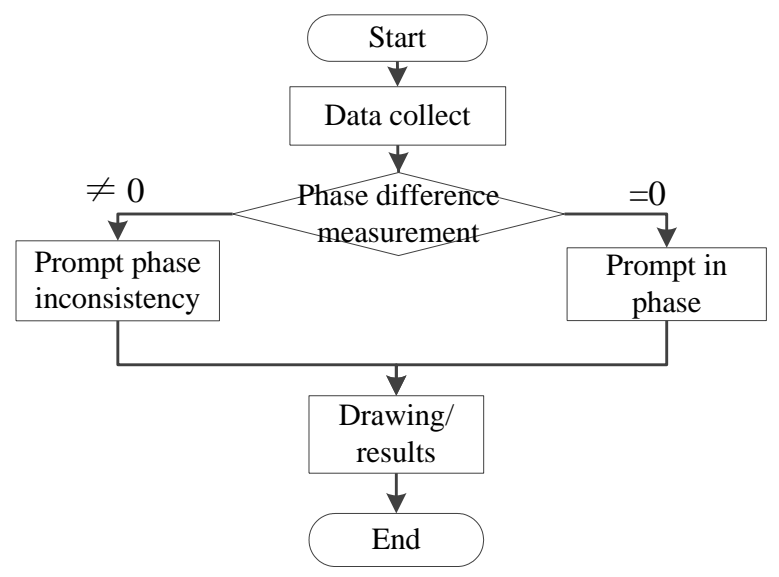

Figure 5. software functional structure design

The entire system software is divided into three modules: acquisition module, process module and a storage module.

Collection function module is to merge unit acquired by the photoelectric conversion device packet analysis, research IEC61850-9-2 communication protocol, and the acquisition of the trigger signal is returned to the embedded computer, thus further utilizing a processing module for processing. The processing module comprising data acquisition module obtained drawing to show the waveform,. For data processing, calculating waveform parameters, the parameters including amplitude, phase difference value and the like. Data processing module includes a waveform display, showing retardation values. Design data storage module, using ADO database technology, the use of SQL database management language, control Access database, the raw data and measurements to be stored for print or duplicate calls.

\section{CONCLUSION}

The paper is for the needs of the substation phasedetection, phase-detection of conventional substation program were introduced, for the problem with intelligent substation phase-detection program is not easy to implement, and analyzes the key technologies required for phase-detection intelligent substation, and gives the specific ways of its realization.

Easy use of phase-detection-based intelligent embedded system, safe and reliable, the production site has a strong practical.

\section{REFERENCE}

[1] GB / T 30155-2013, Intelligent Substation Technical Guidelines $[\mathrm{S}]$.

[2] Yao Xiang, Chen Lin, Dongfeng Yu. Phase-Detection Research on Cable Connected GIS Substation in City[J] , Distribution \& Utilization, 2005,02: 90-92

[3] Du Wen-wei, Yin li-jun, Shen Guang-min, A detection method for the same frequency and phase asynchronous core application [J] Distribution \& Utilization, 2012, 29:. 57-59

[4] Wu Zhen-zhong, Ying Wen-jing, Wang Yao. Cabinet GIS cable terminations dismantling nuclear phase method [J]. Distribution \& Utilization, 2009.05

[5] Du Tong-bo, Li Qing, Li Xiong, Li Guo-shui, Zhou Zhen. Design of embedded systems in phase detection and phase sequence adjustment[J]. Journal of China University of Metrology , ,2012(02) 